\title{
Insights into the aetiology of snoring from observational and genetic investigations in the UK Biobank $(n=408,317)$
}

\author{
Adrián I. Campos, ${ }^{1,2, *}$ Luis M. García-Marín, ${ }^{1,3,}{ }^{*}$ Enda M. Byrne, ${ }^{4}$ Nicholas G. Martin, ${ }^{1}$ Gabriel \\ Cuéllar-Partida, ${ }^{2,5, \ddagger}$ and Miguel E. Rentería ${ }^{1,2, \neq}$
}

1. Genetic Epidemiology Lab, QIMR Berghofer Medical Research Institute, Brisbane QLD Australia

2. Faculty of Medicine, The University of Queensland, Brisbane QLD Australia

3. Tecnológico de Monterrey, Escuela de Ingeniería y Ciencias, Zapopan, Jalisco, México.

4. Institute for Molecular Bioscience, The University of Queensland, Brisbane, QLD, 4072, Australia.

5. University of Queensland Diamantina Institute, Brisbane QLD Australia

*These authors contributed equally to this work; ${ }^{\dagger}$ These authors jointly supervised this work.

Correspondence:

Dr. Miguel E. Rentería, QIMR Berghofer Medical Research Institute, Locked Bag 2000, Royal Brisbane Hospital, Brisbane, Queensland 4029, Australia. Email: miguel.renteria@qimrberghofer.edu.au 


\section{ABSTRACT}

We conducted the largest study of snoring using data from the UK Biobank $(n \sim 408,000$; snorers 152,000). A genome-wide association study (GWAS) identified 42 genome-wide significant loci, with a SNP-based heritability estimate of $\sim 10 \%$ on the liability scale. Genetic correlations with body mass index, alcohol intake, smoking, schizophrenia, anorexia nervosa and neuroticism were observed. Gene-based associations identified 173 genes, including DLEU7, MSRB3 and POC5 highlighting genes expressed in brain, cerebellum, lungs, blood, and oesophagus tissues. We used polygenic scores (PGS) to predict recent snoring and probable obstructive sleep apnoea (OSA) in an independent Australian sample ( $\mathrm{n}$ 8,000). Mendelian randomisation analyses provided evidence that larger whole body fat mass causes snoring. Altogether, our results uncover new insights into the aetiology of snoring as a complex sleep-related trait and its role in health and disease beyond being a cardinal symptom of OSA. 


\section{INTRODUCTION}

Snoring is the vibration of upper airway structures that occurs when the muscles of the airway relax during sleep, thus creating noise as the air passes in and out while breathing. Habitual snoring is common in the population, its overall prevalence increases with age and is higher in males (35-45\%) than females (15-28\%). ${ }^{1}$ Importantly, snoring is a hallmark of OSA, a sleep-related breathing disorder characterised by repeated episodes of complete or partial obstructions of the upper airway during sleep, despite the effort to breathe. ${ }^{1}$ OSA is usually associated with a reduction in blood oxygen saturation, and is often accompanied by associated daytime symptoms, such as excessive daytime sleepiness, fatigue and decreased cognitive function. While the vast majority of patients with OSA exhibit snoring, a minority (20-25\%) of patients with central sleep apnoea do not snore, ${ }^{2}$ and it is estimated that sleep apnoea may occur in as many as 20 to 40 percent of the adult population that are snorers, leaving the remaining $60-80 \%$ of snorers in the category of habitual non-apnoeic benign snorers. Snoring has previously been associated with body mass index $(\mathrm{BMI})^{3,4}$ as well as with risk of cardiovascular disease, such as coronary heart disease and stroke among postmenopausal women. ${ }^{5}$ Twin and family studies have demonstrated the existence of a genetic predisposition to habitual snoring, with heritability estimates suggesting that $18-28 \%$ of variance can be accounted for by genetic factors ${ }^{4,6}$. A proportion of its heritability may be mediated through other heritable lifestyle factors such as smoking and alcohol consumption which can also contribute to snoring. ${ }^{7-9}$

Snoring is known to reduce sleep quality for both snorers and their sleeping partners, ${ }^{10,11}$ reducing energy/vitality and increasing daytime anxiety, ${ }^{12}$ risk of depression, stress, fatigue and sleepiness. ${ }^{11}$ Here, we leverage data from the UK Biobank and an Australian sample of adults, in an effort to characterise the molecular underpinnings of habitual snoring as a complex, polygenic trait, and investigate its relationship to known correlates such as BMI, smoking and alcohol consumption. We also employ statistical genetics methods such as LD score regression and Mendelian randomisation to identify genetically correlated traits with snoring and assess causality. Furthermore, we used our 
bioRxiv preprint doi: https://doi.org/10.1101/808691; this version posted October 17,2019 . The copyright holder for this preprint (which was not certified by peer review) is the author/funder, who has granted bioRxiv a license to display the preprint in perpetuity. It is made available under aCC-BY-NC-ND 4.0 International license.

GWAS results to estimate individual PGS and predict snoring and probable OSA in an independent sample of Australian adults. 


\section{RESULTS}

Snoring prevalence and risk factors. Our population-based discovery sample consisted of 408,317 individuals of white British ancestry from the UK Biobank. Participants in the sample were deemed as snoring 'cases' $(37 \%)$ based on their report that a partner or housemate had complained to the participant about their snoring (see $\underline{\text { Methods }}$ and Table 1). Snoring was significantly associated with age $(\mathrm{OR}=1.011$ [per year, 95\% CI 1.009-1.012] $)$ and, to a greater extent, with sex $\left(\mathrm{OR}_{\text {males }}=2.264\right.$ [2.212-2.316]). The prevalence of sleep apnoea was higher within the snorer group (Table 1). Furthermore, BMI, smoking frequency and alcohol consumption frequency were also associated with snoring (Figure 1a). While snoring prevalence was higher in males, BMI was positively correlated with snoring prevalence in both males and females (Figure 1b). Smoking frequency was positively correlated with snoring prevalence in females, and to a lesser extent in males (Figures 1a and 1c). In contrast, alcohol consumption frequency was correlated with snoring in males, and to a lesser extent in females (Figures 1a and 1d). We further identified other factors such as whole body fat mass and sleep duration that are correlated with snoring (Supplementary Table 1).

Discovery GWAS and SNP heritability. We performed a GWAS of snoring, taken as a dichotomous variable ( $n=408,317$; cases 152,000 ; controls 256,000$)$. After quality control (see

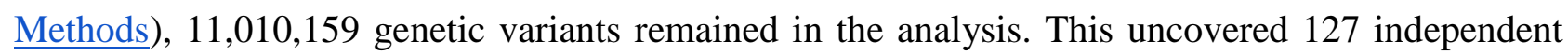
genome-wide significant associations across 41 genomic risk loci (Figure 2 and Supplementary Figure 1). ${ }^{13}$ Annotation for the top 15 risk loci is shown in Table 2, and a list of all genomic risk loci is given in Supplementary File 1. The overall SNP heritability on the liability scale $\left(h^{2}{ }_{S N P}\right)$ was $9.9 \%$ (S.E. $=0.39 \%)$.

Genetic correlations. The trait that showed the highest genetic correlation with habitual snoring was self reported sleep apnoea $\left(r_{G}=0.78\right.$ S.E. $=0.17$, p-value $=3 \times 10^{-05}$; Supplementary File 2). Other genetically correlated traits with snoring included BMI, whole body fat mass, sodium in urine, mood 
swings, coronary artery disease, alcohol intake frequency, pulse rate, current tobacco smoking, heart disease, lung cancer, the ratio between forced expiratory volume in 1 second (FEV1) and forced vital capacity (FVC), neuroticism, subjective wellbeing and heart rate, among others. Traits showing a negative genetic correlation with snoring included schizophrenia, FVC, FEV1, fluid intelligence score, educational attainment, age at menarche, mean accumbens volume and anorexia nervosa. Overall, traits related to body-mass index, risk for psychiatric disease, lung function and heart disease were among those with the strongest evidence of association (Figure 3 and Supplementary File 2). Notably, pulse rate, whole body fat mass and BMI were also phenotypically associated with snoring in this sample (see above and Supplementary Table 1).

Sensitivity Analysis. We performed a follow-up sensitivity GWAS for snoring including BMI as a covariate to explore the effects of BMI on associated variants. The results revealed 97 genome-wide significant SNPs across 34 genomic risk loci (Figure 2) with overall SNP heritability on the liability scale $\left(h^{2}{ }_{S N P}\right)$ of $8.67 \%($ S.E. $=0.39)$ (Table 3). Traits that remained genetically correlated with snoring after adjusting for BMI included schizophrenia, educational attainment, sodium in urine and sleep related traits such as daytime dozing, sleep apnoea and excessive daytime sleepiness (Supplementary File 3). The genetic correlation between both the adjusted and unadjusted GWAS was high $\left(r_{\mathrm{G}}=0.923\right.$, S.E. $=0.003$, p-value $\left.=1 \times 10^{-300}\right)$ suggesting that a considerable amount of snoring predisposition is not fully explained by BMI.

Positional, eQTL and gene-based test prioritisation. To gain insights into the functional consequences of individual genome-wide significant variants, we used positional and eQTL mapping as well as genome-wide gene-based association analyses. From positional and eQTL mapping, we identified 149 protein coding genes mapping to a genome-wide significant SNP. The nearest genes to the top signals included DLEU7 on chromosome 13, and MSRB3 on chromosome 12. In addition to DLEU7 and MSRB3, other compelling genes (prioritised by positional or eQTL mapping) for 
snoring included BCL11B, FTO, SMG6, ROBO2, NSUN3, SNAP91 and BCL2, which have previously been associated with smoking; ${ }^{14,15} B L C 11 B, F T O^{16}, R N A 5 S P 471^{16,17}$ and SND1 and NSUN3, previously associated with alcohol consumption; ${ }^{14,16-18}$ FTO and SND1, associated with coffee consumption; ${ }^{19}$ LMO4 associated with insomnia; ${ }^{20}$ and RNA5SP471 with narcolepsy. ${ }^{20,21}$ Also, ROBO2, previously associated with chronotype, ${ }^{22,23}$ and multiple genes (DLEU7, MSRB3, FTO, ANAPC4, SMG6, SND1, SIM1, KCNQ5, CEP120, MACF1, SNAP91 and BCL2) previously associated with musculoskeletal traits such as height and heel bone mineral density (Supplementary File 1 and Supplementary File 2). ${ }^{24-27}$ Genome-wide gene-based association analysis identified 179 genes associated with snoring beyond genome wide significance $(\mathrm{p}<2.636 \mathrm{e}-6$; bonferroni corrected threshold for 18,971 tested genes) several of which were consistent with the mapped genes. After adjusting for BMI, 104 protein coding genes were identified mapping to a genome-wide significant SNP from the positional and eQTL mapping while 120 genes remained significantly associated with snoring, including both MSRB3 and DLEU7 (See Supplementary Figure 2 and Supplementary Files 2 and 3 ). eQTL data obtained from GTEx and mapped with FUMA highlighted significant SNPs that were associated with the expression of genes in several tissues including lungs, blood, oesophagus, breast mammary, tibial nerve, and several areas of the brain, such as the cerebellum and hippocampus (Supplementary Figure 3 and Supplementary File 2). In summary, many of the mapped genes for snoring have been previously associated with other traits and diseases, primarily grouped into cardiometabolic, cognitive/neurological, respiratory and psychiatric (Figure 4 and Supplementary File 1).

To further assess whether significant genes converged in functional gene sets and pathways, we conducted gene-set enrichment analyses of tissue expression data (Supplementary Figure 3a). Genes expressed in blood vessel and tibial artery tissue were associated with snoring, even after adjusting for BMI (Supplementary Figure 3b). Given these associations, and an observed genetic correlation 
between snoring and pulse rate $\left(r_{\mathrm{G}}=0.106\right.$, S.E. $=0.03$, p-value $\left.=0.001\right)$, we conducted two sample Generalised Summary-data-based Mendelian Randomisation (GSMR) ${ }^{28}$ to test for a possible causal relationship. The analysis suggested a one-way causal relationship in which snoring increased pulse rate (Supplementary Figure 4). We further explored the association between snoring and BMI or whole body fat mass. GSMR results suggested a bidirectional causal relationship, with snoring exerting a causal effect on BMI, but also BMI exerting a causal effect on snoring. However, a oneway causal relationship was seen for whole body fat mass causing snoring (Supplementary Figure 4). To control for possible confounding due to sample overlap, we repeated the Mendelian randomisation analyses using sex-stratified GWAS results (see Methods). This confirmed the causal associations between BMI or whole body fat mass causing snoring, while all other associations did not retain statistical significance after controlling for multiple testing (Figure 5 and Supplementary Table 2).

Sex-stratified GWAS. Given the higher prevalence of snoring in males, we conducted GWAS analyses stratified by sex. These analyses identified 4 and 25 genome-wide significant SNPs for snoring in males and females, respectively. SNP heritability on the liability scale $\left(h^{2}{ }_{S N P}\right)$ was $8.77 \%$ $($ S.E. $=0.54 \%)$ and $12.42 \%$ (S.E.=0.57\%) respectively for males and females (Table 3). In the sensitivity analyses, SNP heritability $\left(h^{2}{ }_{S N P}\right)$ was slightly lower after adjusting for BMI in both males $7.72 \%($ S.E. $=0.56 \%)$ and females $10.85 \%($ S.E. $=0.54 \%)(\underline{\text { Table 3) }}$. The cross-sex genetic correlation was high $\left(r_{\mathrm{G}}=0.914\right.$, S.E. $=0.033$, p-value $\left.=1.91 \times 10^{-160}\right)$ and effect sizes and directions for top hits were highly consistent in both the male and female samples (Supplementary File 1 and Supplementary Figure 5).

Polygenic scores and prediction on an independent target sample. We used the discovery GWAS summary statistics to derive PGS in an independent target sample from the Australian Genetics of Depression Study (AGDS). The prevalence of self-reported recent snoring was 32\%, with a higher 
prevalence in males than females (43.2\% and $28.1 \%$ respectively). PGS for snoring were significantly associated with recent snoring for all but one $(\mathrm{p}<=5 \mathrm{e}-8)$ of the $\mathrm{p}$-value inclusion thresholds (Figure $\underline{6}$ and Supplementary Figure 6). Participants in the highest snoring PGS decile had around twice the odds of reporting recent snoring and choking or struggling for breath during sleep (i.e. probable OSA; sample prevalence $=8.2 \%$ ) compared to those on the lowest decile (Figure 6a). Furthermore, the PGS showed a stronger association with increasing frequency of snoring severity (igure 6b). Finally, we showed that the snoring PGS explained a significant amount of variance in recent snoring (Figure $\underline{6 c)}$. 


\section{DISCUSSION}

This study advances our understanding of the aetiology and genetic architecture of snoring. Overall, snoring prevalence was higher in males than in females, having a strong and positive correlation with BMI, tobacco smoking and alcohol consumption in both sexes. The effects of BMI, smoking and alcohol consumption have been previously reported in other studies. ${ }^{7,29,30}$ In our study, tobacco smoking displayed a stronger association with snoring in females compared to males, a result consistent with an observations in an independent sample of $\sim 15,000$ Europeans published in $2004 .{ }^{30}$ Previous studies provided evidence that women might have a greater susceptibility to chronic obstructive pulmonary disease after smoking ${ }^{31}$ and a greater bronchial hyperresponsiveness after methacholine challenge. ${ }^{32}$ These results suggest that smoking might be associated with snoring through an increased inflammatory response and irritation of the airways, thus having a larger effect size on females, compared to males. Conversely, our study indicates that the frequency of alcohol consumption has a stronger influence on snoring in males, compared to females. This is consistent with a previous study which failed to identify an association between alcohol consumption and snoring in females but did so in males. ${ }^{33}$ Our results strengthen the evidence pointing to alcohol as a risk factor for snoring and sleep apnoea, potentially through a weakening (relaxation) effect in the jaw and pharyngeal muscles. ${ }^{34}$ Future studies should leverage statistical genetics methods such as polygenic scoring or Mendelian randomisation to further characterise the role of smoking and alcohol-related phenotypes in snoring.

The sex differences described above motivated us to perform sex-stratified GWAS. The larger sample size of the female subgroup conferred more power to detect genetic associations in our analyses. The observed high cross-sex genetic correlation, and a high concordance in effect size and direction amongst the top hits suggests that differences in sex-stratified GWAS might be simply due to power differences between the male and female subsamples rather than the existence of sex-specific genetic effects. 
Top genes identified from gene-based test analysis for snoring included DLEU7 and MSRB3. Previous reports have associated DLEU7 with heel bone mineral density, ${ }^{24,35} \mathrm{BMI},{ }^{36,37}$ height, ${ }^{38,39}$ cardiovascular diseases, ${ }^{40}$ systolic blood pressure ${ }^{40}$ and pulmonary function decline (forced expiratory volume). ${ }^{41}$ The association between snoring genes and heel bone mineral density could be mediated by BMI due to the association between BMI and bone density documented previously. ${ }^{42}$ $M S R B 3$ plays a relevant role in protein and lipid metabolism pathways ${ }^{43}$ and has been associated with hippocampal volume, ${ }^{44-46}$ lung function,,${ }^{40,47,48}$ Alzheimer's disease, ${ }^{49,50}$ brain injuries, ${ }^{51}$ novelty seeking, ${ }^{52}$ deafness ${ }^{53}$ and height. ${ }^{40}$ These results could be consistent with the fact that severe snoring may incur in nocturnal oxygen desaturation, ${ }^{54}$ diminishing neuropsychological functions and in some cases, resulting in tissue damage ${ }^{55}$ and contributing to impairment of memory consolidation processes ${ }^{56}$ However, more research is needed in order to test this hypothesis.

Genetic correlations between snoring and a variety of traits and diseases were identified. The trait with the highest genetic correlation with snoring is sleep apnoea, which is consistent with loud snoring being a diagnostic criterion for OSA. This observation is also remarkable given the small sample size (less than 2000 cases) and therefore reduced power (no genome-wide hits) that the GWAS for self-reported sleep apnoea has in the UK Biobank. Our analyses suggest that a GWAS for snoring captures a substantial portion of the genetic contribution to sleep apnoea, highlighting the importance of studying symptoms on a subclinical threshold, an approach that has already proven useful at understanding the heterogeneity of other complex traits such as depression and neuroticism. ${ }^{57,58}$ Our study will enable future efforts aimed at understanding the underlying genetic architecture of OSA using multivariate statistical genetic approaches.

We also observed moderate correlations with BMI, obesity and whole body fat mass. Other relevant correlations included lung function, neurological, cardiovascular and psychiatric diseases. The high 
genetic correlation between snoring and snoring adj. BMI $\left(r_{\mathrm{G}}=0.923\right.$, S.E. $=0.003$, p-value $\left.=1 \times 10^{-300}\right)$ supports the idea that the genetic architecture of snoring cannot be explained simply by BMI. This highlights the importance of studying snoring as a trait in its own, which might open new opportunities to the understanding of highly related sleeping disorders, including OSA, as well as its relationship with other traits and diseases.

Our initial Mendelian randomisation results suggested a mutual causal relationship between BMI and snoring, but only a one-way causal relationship of whole body fat mass causing snoring. We hypothesise BMI to be more heterogeneous and potentially more pleiotropic than whole body fat mass. In fact Mendelian randomisation is known to be confounded by pleiotropy. ${ }^{59}$ Interestingly, a one-way causal relationship between snoring and pulse rate, which survived adjustment for BMI, was identified. Nonetheless, this association did not reach statistical significance when accounting for sample overlap (i.e. sex-stratified GSMR). The only causal associations that survived this were BMI or whole body fat mass causing snoring. The significantly lower number of instruments available for snoring as an exposure (Supplementary Table 2) makes it hard to assess whether the results were due to a lack of power or a lack of true causal effect. Future efforts could leverage novel statistical genetics methods that use all the GWAS results to test whether the associations observed could be explained by a causality rather than pleiotropy. ${ }^{60}$

Finally, we assessed the validity of our GWAS results by using genetic scoring on an independent sample of Australian adults with data on recent snoring. Our successful prediction of snoring using PGS supports the external validity of our genetic association results. Remarkably, we predicted probable OSA using a snoring derived PGS. Thus, investigating the aetiology of snoring could also help uncover the aetiology and genetic architecture of OSA, a task that has proved difficult and challenging ${ }^{61}$. Future efforts could assess whether the utility of snoring derived PGS as an addition 
to the current battery of tests used to more accurately diagnose OSA, ${ }^{62}$ particularly given the issue of potential OSA underdiagnosis. ${ }^{63,64}$

Our results highlight the relevance of studying snoring as an independent trait, and provide important insights into its aetiology and genetic architecture. This study represents the largest genetic investigation of snoring to date. However, some limitations must be acknowledged. Analyses used self-reported snoring with the item relying on a partner or close friend complaining about the participant's snoring. Thus, the case definition might be subject to participant specific recall and subjective biases. Nonetheless, we hypothesize that this limitation might result in the inclusion of some cases as controls (i.e. snoring participants living alone) and therefore bias our results toward the null rather than creating false positives. To avoid confounding due to population stratification, we only included samples of European ancestry in our analyses. This is particularly important given reports of ethnic differences associated with snoring prevalences. ${ }^{65}$ Nonetheless, excluding other populations can limit the generalisability of these results outside the populations studied. As previously discussed, we cannot identify which, if any, sex-specific genetic observations (e.g. differences in SNP heritability) are due to true genetic effects rather than power differences between the samples. Finally, the fact that PGS for snoring predicted less than one percent of the variance on recent snoring suggest that the GWAS is still underpowered ${ }^{66}$. The heritability for snoring in twin studies is estimated in the range of 18 to $28 \%$, although some of the missing heritability for snoring may come from dominant genetic effects, it is likely that an increased power and studying rare variants ${ }^{67}$ yields more powered genetic predictors. 


\section{CONCLUSIONS}

In summary, we provide insights into the aetiology of snoring, its risk factors and genetic underpinnings. Our observational analyses showed a higher prevalence of snoring in males compared to females, and effects of age, BMI, smoking and alcohol consumption. In addition, tobacco smoking showed a higher effect on snoring prevalence in females compared to males while alcohol consumption displayed a higher effect on snoring prevalence in males compared to females. GWAS identified 127 genome-wide significant associations across 41 genomic risk loci with $h^{2}{ }_{S N P}=9.9 \%$. We found no evidence for sex-specific genetic factors, and showed that most of the SNP heritability identified is not simply due to BMI. We also found evidence of a causal relationship from BMI or whole body fat mass to snoring. We further found evidence of genetic overlap between snoring and other cardiometabolic, respiratory, neurological and psychiatric traits. Finally, we used the GWAS summary statistics to derive individual PGS and predict both recent snoring and probable OSA in an independent sample of Australian adults, thus confirming the relevance of snoring as a sleep-related complex trait. Future studies should aim at leveraging powered GWAS on alcohol and tobacco behaviours to assess whether they are truly causal of snoring, and to assess the amount of shared genetic overlap between OSA and habitual snoring, as the latter may serve to boost the power of obstructive sleep apnoea genetic studies. 


\section{METHODS}

\section{Discovery sample and phenotypic information.}

Participants included in the present study were of European ancestry from the UK Biobank. Briefly, this resource recruited participants between 2006 and 2010 to assess lifestyle, anthropometric and health related variables. Participants self-reported on sleep-related traits. Snoring was assessed as a single item (Field-ID: 1210): "Does your partner or a close relative or friend complain about your snoring?" This question could be answered with "Yes", "No", "Don't know", or "Prefer not to answer". We excluded participants whose answers were "Don't know" $(n=29,309)$ or "Prefer not to answer" $(n=6,854)$ from our analyses. (Supplementary Table 3 shows the total sample size for each GWAS, including sensitivity and sex-stratified analyses). OSA cases were determined on the basis of either ICD-10 diagnosis code or self-report of sleep apnoea diagnosis in the UK Biobank.

\section{Data extraction and statistical analyses}

Raw data was extracted from the UK Biobank under Application Number 25331. For a description of the field codes and instances used refer to Supplementary Table 4. Data was re-coded to remove missing data and uninformative responses (e.g. "I don’t know" or "I would rather not answer"). Phenotype derived estimates such as prevalence and associations between variables were calculated using python. Libraries such as numpy (https://docs.scipy.org/doc/numpy/user/) and scipy (https://docs.scipy.org/doc/) were used for descriptive statistics and statsmodels (http://conference.scipy.org/proceedings/scipy2010/pdfs/seabold.pdf) was used to build logistic regression models to assess correlates of snoring and calculate odds ratios. Snoring prevalence stratified plots (e.g. Figure 1) were performed using seaborn v0.9.0. Confidence intervals were calculated using a bootstrapping procedure generating 1000 pseudo-replicates of the data.

\section{Genetic association analyses and quality control.}


Discovery GWAS. Analyses were performed for both sex stratified and full sample using the BOLT-

LMM software tool. We used a strict quality control (QC) procedure corresponding to minor allele frequency $(\mathrm{MAF}>=0.005)$ and imputation quality $(>=0.60)$.

Sensitivity analyses. Given the strong correlation between snoring and BMI, we carried out GWAS for snoring using BMI as a covariate (snoring adj. BMI) with BOLT-LMM software with a quality control corresponding to minor allele frequency $(\mathrm{MAF}>=0.005)$ and imputation quality $(>=0.60)$.

\section{Post-GWAS Annotation and Functional Mapping.}

SNP annotation was conducted using the FUMA platform. Risk loci are defined as up to $250 \mathrm{~kb}$ based on the most right and left SNPs from each LD block. Gene based tests were performed using Multimarker Analysis of GenoMic Annotation (MAGMA) as implemented on the FUMA platform, which provides aggregate association p-values based on all variants located within a gene and its regulatory region. We used the GWAS summary statistics to conduct a MAGMA ${ }^{68}$ analysis in the FUMA ${ }^{13}$ platform (https://fuma.ctglab.nl/). This analysis includes a gene-based test to detect significant SNPs associated with snoring. The prioritised genes based on positional and eQTL mapping were further used to perform gene-set enrichment analysis against the traits available in the GWAS catalog. Furthermore, we used FUMA to perform tissue enrichment analysis, based on data from the Genotype-Tissue Expression (GTEx) project (https://gtexportal.org/home/documentationPage).

\section{Genetic correlation analyses.}

We performed genetic correlation analyses to estimate genetic correlations between the discovery, sensitivity and sex-stratified snoring GWAS summary statistics using LD score regression as implemented in the Complex Trait Genomics Virtual Lab (CTG-VL, http://genoma.io). Further, in order to uncover genetically correlated traits with snoring, genetic correlation analyses using LD Score Regression (LDSC) were performed on the platforms CTG-VL and LDHub 
(http://ldsc.broadinstitute.org/ldhub/), which aggregate summary statistics for GWAS on hundreds of traits.

\section{Mendelian randomisation}

Mendelian randomisation (MR) is a method in which genetic variants (e.g. single nucleotide polymorphisms, SNPs) are used as instrumental variables to determine causal relationships between traits, environmental exposures, biomarkers, or disease outcomes ${ }^{69}$; to satisfy the conditions for MR, it is not required to identify the actual causal variant because a marker in Linkage Disequilibrium (LD) with the causal variant can serve as a proxy instrument ${ }^{70}$. Moreover, in order to draw conclusions in regard with casual effects, three relevant assumptions must be taken into consideration: (I) genetic variants must be associated with the exposure of interest; (II) genetic variants must not be confounded; (III) genetic variants must be independent of the outcome through other mechanisms. ${ }^{71}$ We used Generalised Summary-data-based Mendelian Randomisation $(\mathrm{GSMR})^{70}$, an approach that leverages the usage of multiple independent variables (SNPs) strongly associated with the outcome to overcome these assumptions, as implemented in the CTG -VL (http://genoma.io). We used GSMR to assess causal relationships between snoring and: BMI, whole body fat mass and pulse rate using our results and existing summary statistics for these traits. To avoid possible confounding from sample overlap, we performed GSMR using the summary statistics derived from sex-stratified GWAS. For example, the female snoring GWAS results were used as exposure while the male pulse rate GWAS results were used for the outcome.

\section{Target sample and polygenic scoring}

In order to quantify for the cumulative genetic associations for snoring, we calculated PGS using a clumping + thresholding approach. Study description and sample characteristics of the Australian Genetics of Depression study is available elsewhere. ${ }^{72}$ Genotyping was conducted using the Illumina Infinium Global Screening Array platform and genotype imputation using the Haplotype Reference 
Consortium's reference panel in the Michigan Imputation Server ${ }^{73}$ was carried out after performing standard quality control procedures. Briefly, for PGS estimation, we excluded indel, strand ambiguous- and low $(\mathrm{R} 2<0.6)$ imputation quality-variants. The most significant SNPs were selected using a conservative clumping procedure (PLINK1.974; $1=1, \mathrm{p} 2=1, \mathrm{r} 2=0.1, \mathrm{~kb}=10000)$ to correct for inflation arising from linkage disequilibrium (LD). Eight PGS were calculated using different pvalue thresholds $(\mathrm{p}<5 \mathrm{x} 10-8, \mathrm{p}<1 \mathrm{x} 10-5, \mathrm{p}<0.001, \mathrm{p}<0.01, \mathrm{p}<0.05, \mathrm{p}<0.1, \mathrm{p}<0.5, \mathrm{p}<1)$ as criteria for SNP inclusion on the PGS calculation. PGS were calculated by multiplying the effect size of a given SNP by the imputed number of copies (using dosage probabilities) of the effect allele present in an individual. Finally, the SNP dosage effects were summed across all loci per individual. To assess the association between the PGS and snoring and probable OSA, we employed a logistic regression (python statsmodels). The target sample for snoring was a subset $(\mathrm{n}=9,026)$ of the Australian Genetics of Depression Study (AGDS) with data on recent snoring collected through the self-reported item: "During the last month, on how many nights or days per week have you had or been told you had loud snoring". The item for probable OSA was: "During the last month, on how many nights or days per week have you had or been told your breathing stops or you choke or struggle for breath". For both items a positive response was considered from 1-2 times per week up to 5-7 times per week and the answer Rarely, less than once a week was excluded. Only a subset $(\mathrm{n} \sim 8,000)$ of highly unrelated individuals (genetic relatedness $<0.05$ ) were included in the analyses.

\section{REFERENCES}

1. Main, C. et al. Surgical procedures and non-surgical devices for the management of nonapnoeic snoring: a systematic review of clinical effects and associated treatment costs. Health Technol. Assess. 13, iii, xi-xiv, 1-208 (2009).

2. Bauters, F. A. et al. Phenotype and Risk Burden of Sleep Apnea. Hypertension HYPERTENSIONAHA11913452 (2019).

3. Ferini-Strambi, L. et al. Snoring in twins. Respir. Med. 89, 337-340 (1995). 
4. Jennum, P., Hein, H. O., Suadicani, P., Sørensen, H. \& Gyntelberg, F. Snoring, family history, and genetic markers in men. The Copenhagen Male Study. Chest 107, 1289-1293 (1995).

5. Sands, M. et al. Self-reported snoring and risk of cardiovascular disease among postmenopausal women (from the Women's Health Initiative). Am. J. Cardiol. 111, 540-546 (2013).

6. Carmelli, D., Bliwise, D. L., Swan, G. E. \& Reed, T. Genetic factors in self-reported snoring and excessive daytime sleepiness: a twin study. Am. J. Respir. Crit. Care Med. 164, 949-952 (2001).

7. Katila, M., Saarenpää-Heikkilä, O., Saha, M.-T., Vuorela, N. \& Paavonen, E. J. Parental reports showed that snoring in infants at three and eight months associated with snoring parents and smoking mothers. Acta Paediatr. 108, 1686-1694 (2019).

8. Wei Y. X. et al. [Epidemiological characteristics and correlated factors of habitual snoring among Chinese aged 30 to 79 year-old]. Zhonghua Liu Xing Bing Xue Za Zhi 40, 917-923 (2019).

9. Morales-Muñoz, I., Koskinen, S. \& Partonen, T. The effects of seasonal affective disorder and alcohol abuse on sleep and snoring functions in a population-based study in Finland. J. Sleep Res. 27, e12611 (2018).

10. Blumen, M. et al. Effect of sleeping alone on sleep quality in female bed partners of snorers. Eur. Respir. J. 34, 1127-1131 (2009).

11. Beninati, W., Harris, C. D., Herold, D. L. \& Shepard, J. W., Jr. The effect of snoring and obstructive sleep apnea on the sleep quality of bed partners. Mayo Clin. Proc. 74, 955-958 (1999).

12. Doherty, L. S., Kiely, J. L., Lawless, G. \& McNicholas, W. T. Impact of nasal continuous positive airway pressure therapy on the quality of life of bed partners of patients with obstructive sleep apnea syndrome. Chest 124, 2209-2214 (2003).

13. Watanabe, K., Taskesen, E., van Bochoven, A. \& Posthuma, D. Functional mapping and 
annotation of genetic associations with FUMA. Nat. Commun. 8, 1826 (2017).

14. Liu, M. et al. Association studies of up to 1.2 million individuals yield new insights into the genetic etiology of tobacco and alcohol use. Nat. Genet. 51, 237-244 (2019).

15. Justice, A. E. et al. Genome-wide meta-analysis of 241,258 adults accounting for smoking behaviour identifies novel loci for obesity traits. Nat. Commun. 8, 14977 (2017).

16. Kranzler, H. R. et al. Genome-wide association study of alcohol consumption and use disorder in 274,424 individuals from multiple populations. Nat. Commun. 10, 1499 (2019).

17. Whitfield, J. B. et al. Biomarker and Genomic Risk Factors for Liver Function Test Abnormality in Hazardous Drinkers. Alcohol. Clin. Exp. Res. 43, 473-482 (2019).

18. Karlsson Linnér, R. et al. Genome-wide association analyses of risk tolerance and risky behaviors in over 1 million individuals identify hundreds of loci and shared genetic influences. Nat. Genet. 51, 245-257 (2019).

19. Zhong, V. W. et al. A genome-wide association study of bitter and sweet beverage consumption. Hum. Mol. Genet. 28, 2449-2457 (2019).

20. Lane, J. M. et al. Biological and clinical insights from genetics of insomnia symptoms. Nat. Genet. 51, 387-393 (2019).

21. Luca, G. et al. Clinical, polysomnographic and genome-wide association analyses of narcolepsy with cataplexy: a European Narcolepsy Network study. J. Sleep Res. 22, 482-495 (2013).

22. Jansen, P. R. et al. Genome-wide analysis of insomnia in 1,331,010 individuals identifies new risk loci and functional pathways. Nat. Genet. 51, 394-403 (2019).

23. Jones, S. E. et al. Genome-wide association analyses of chronotype in 697,828 individuals provides insights into circadian rhythms. Nat. Commun. 10, 343 (2019).

24. Kim, S. K. Identification of 613 new loci associated with heel bone mineral density and a polygenic risk score for bone mineral density, osteoporosis and fracture. PLoS One 13, e0200785 (2018). 
25. Kim, K. et al. High-density genotyping of immune loci in Koreans and Europeans identifies eight new rheumatoid arthritis risk loci. Ann. Rheum. Dis. 74, e13 (2015).

26. Morris, J. A. et al. An atlas of genetic influences on osteoporosis in humans and mice. Nat. Genet. 51, 258-266 (2019).

27. Medina-Gomez, C. et al. Life-Course Genome-wide Association Study Meta-analysis of Total Body BMD and Assessment of Age-Specific Effects. Am. J. Hum. Genet. 102, 88-102 (2018).

28. Zhu, Z. et al. Causal associations between risk factors and common diseases inferred from GWAS summary data. Nat. Commun. 9, 224 (2018).

29. Nagayoshi, M. et al. Risk factors for snoring among Japanese men and women: a communitybased cross-sectional study. Sleep Breath. 15, 63-69 (2011).

30. Franklin, K. A. et al. The influence of active and passive smoking on habitual snoring. Am. J. Respir. Crit. Care Med. 170, 799-803 (2004).

31. Jordan, R. E. et al. Sex, susceptibility to smoking and chronic obstructive pulmonary disease: the effect of different diagnostic criteria. Analysis of the Health Survey for England. Thorax 67, 600-605 (2012).

32. Leynaert, B., Bousquet, J., Henry, C., Liard, R. \& Neukirch, F. Is bronchial hyperresponsiveness more frequent in women than in men? A population-based study. Am. J. Respir. Crit. Care Med. 156, 1413-1420 (1997).

33. Peppard, P. E., Austin, D. \& Brown, R. L. Association of alcohol consumption and sleep disordered breathing in men and women. J. Clin. Sleep Med. 3, 265-270 (2007).

34. Issa, F. G. \& Sullivan, C. E. Alcohol, snoring and sleep apnea. J. Neurol. Neurosurg. Psychiatry 45, 353-359 (1982).

35. Kemp, J. P. et al. Identification of 153 new loci associated with heel bone mineral density and functional involvement of GPC6 in osteoporosis. Nat. Genet. 49, 1468-1475 (2017).

36. Shungin, D. et al. New genetic loci link adipose and insulin biology to body fat distribution. Nature 518, 187-196 (2015). 
37. Tachmazidou, I. et al. Whole-Genome Sequencing Coupled to Imputation Discovers Genetic Signals for Anthropometric Traits. Am. J. Hum. Genet. 100, 865-884 (2017).

38. Wood, A. R. et al. Defining the role of common variation in the genomic and biological architecture of adult human height. Nat. Genet. 46, 1173-1186 (2014).

39. Lango Allen, H. et al. Hundreds of variants clustered in genomic loci and biological pathways affect human height. Nature 467, 832-838 (2010).

40. Kichaev, G. et al. Leveraging Polygenic Functional Enrichment to Improve GWAS Power. Am. J. Hum. Genet. 104, 65-75 (2019).

41. Imboden, M. et al. Genome-wide association study of lung function decline in adults with and without asthma. J. Allergy Clin. Immunol. 129, 1218-1228 (2012).

42. Salamat, M. R., Salamat, A. H., Abedi, I. \& Janghorbani, M. Relationship between Weight, Body Mass Index, and Bone Mineral Density in Men Referred for Dual-Energy X-Ray Absorptiometry Scan in Isfahan, Iran. J. Osteoporos. 2013, 205963 (2013).

43. Kim, H.-Y. \& Gladyshev, V. N. Methionine sulfoxide reduction in mammals: characterization of methionine-R-sulfoxide reductases. Mol. Biol. Cell 15, 1055-1064 (2004).

44. van der Meer, D. et al. Brain scans from 21,297 individuals reveal the genetic architecture of hippocampal subfield volumes. Mol. Psychiatry (2018). doi:10.1038/s41380-018-0262-7

45. Hibar, D. P. et al. Novel genetic loci associated with hippocampal volume. Nat. Commun. 8, 13624 (2017).

46. Bis, J. C. et al. Common variants at $12 \mathrm{q} 14$ and $12 \mathrm{q} 24$ are associated with hippocampal volume. Nat. Genet. 44, 545-551 (2012).

47. Wain, L. V. et al. Genome-wide association analyses for lung function and chronic obstructive pulmonary disease identify new loci and potential druggable targets. Nat. Genet. 49, 416-425 (2017).

48. Shrine, N. et al. New genetic signals for lung function highlight pathways and chronic obstructive pulmonary disease associations across multiple ancestries. Nat. Genet. 51, 481- 
493 (2019).

49. Gabbita, S. P., Aksenov, M. Y., Lovell, M. A. \& Markesbery, W. R. Decrease in peptide methionine sulfoxide reductase in Alzheimer's disease brain. J. Neurochem. 73, 1660-1666 (1999).

50. Adams, S. L. et al. Methionine Sulfoxide Reductase-B3 (MsrB3) Protein Associates with Synaptic Vesicles and its Expression Changes in the Hippocampi of Alzheimer's Disease Patients. J. Alzheimers. Dis. 60, 43-56 (2017).

51. Conner, S. C. et al. Methionine Sulfoxide Reductase-B3 Risk Allele Implicated in Alzheimer's Disease Associates with Increased Odds for Brain Infarcts. J. Alzheimers. Dis. 68, 357-365 (2019).

52. Service, S. K. et al. A genome-wide meta-analysis of association studies of Cloninger's Temperament Scales. Transl. Psychiatry 2, e116 (2012).

53. Ahmed, Z. M. et al. Functional null mutations of MSRB3 encoding methionine sulfoxide reductase are associated with human deafness DFNB74. Am. J. Hum. Genet. 88, 19-29 (2011).

54. Block, A. J., Hellard, D. W. \& Cicale, M. J. Snoring, nocturnal hypoxemia, and the effect of oxygen inhalation. Chest 92, 411-417 (1987).

55. Kou, Z., Ye, Y. \& Haacke, E. M. Evaluating the Role of Reduced Oxygen Saturation and Vascular Damage in Traumatic Brain Injury Using Magnetic Resonance Perfusion-Weighted Imaging and Susceptibility-Weighted Imaging and Mapping. Top. Magn. Reson. Imaging 24, 253-265 (2015).

56. Rasch, B. \& Born, J. About Sleep's Role in Memory. Physiological Reviews 93, 681-766 (2013).

57. Thorp, J. G. et al. Genetic heterogeneity in self-reported depressive symptoms identified through genetic analyses of the PHQ-9. Psychol. Med. 1-12

58. Nagel, M., Watanabe, K., Stringer, S., Posthuma, D. \& van der Sluis, S. Item-level analyses reveal genetic heterogeneity in neuroticism. Nat. Commun. 9, 905 (2018). 
59. Verbanck, M., Chen, C.-Y., Neale, B. \& Do, R. Detection of widespread horizontal pleiotropy in causal relationships inferred from Mendelian randomization between complex traits and diseases. Nat. Genet. 50, 693-698 (2018).

60. O’Connor, L. J. \& Price, A. L. Distinguishing genetic correlation from causation across 52 diseases and complex traits. Nat. Genet. 50, 1728-1734 (2018).

61. Pham, L. V. \& Polotsky, V. Y. Genome-Wide Association Studies in Obstructive Sleep Apnea. Will We Catch a Black Cat in a Dark Room? Am. J. Respir. Crit. Care Med. 194, 789791 (2016).

62. McNicholas, W. T. Diagnosis of obstructive sleep apnea in adults. Proc. Am. Thorac. Soc. 5, $154-160$ (2008).

63. Costa, L. E. et al. Potential underdiagnosis of obstructive sleep apnoea in the cardiology outpatient setting. Heart 101, 1288-1292 (2015).

64. Motamedi, K. K., McClary, A. C. \& Amedee, R. G. Obstructive Sleep Apnea: A Growing Problem. Ochsner J. 9, 149 (2009).

65. Goldstein, N. A. et al. Racial/ethnic differences in the prevalence of snoring and sleep disordered breathing in young children. J. Clin. Sleep Med. 7, 163-171 (2011).

66. Dudbridge, F. Power and predictive accuracy of polygenic risk scores. PLoS Genet. 9, e1003348 (2013).

67. Wainschtein, P. et al. Recovery of trait heritability from whole genome sequence data. bioRxiv 588020 (2019). doi:10.1101/588020

68. de Leeuw, C. A., Mooij, J. M., Heskes, T. \& Posthuma, D. MAGMA: generalized gene-set analysis of GWAS data. PLoS Comput. Biol. 11, e1004219 (2015).

69. Swerdlow, D. I. et al. Selecting instruments for Mendelian randomization in the wake of genome-wide association studies. Int. J. Epidemiol. 45, 1600-1616 (2016).

70. Davey Smith, G. \& Hemani, G. Mendelian randomization: genetic anchors for causal inference in epidemiological studies. Hum. Mol. Genet. 23, R89-98 (2014). 
71. Benn, M. \& Nordestgaard, B. G. From genome-wide association studies to Mendelian randomization: novel opportunities for understanding cardiovascular disease causality, pathogenesis, prevention, and treatment. Cardiovasc. Res. 114, 1192-1208 (2018).

72. Byrne, E. M. et al. The Australian Genetics of Depression Study: Study Description and Sample Characteristics. doi:10.1101/626762

73. Das, S. et al. Next-generation genotype imputation service and methods. Nat. Genet. $\mathbf{4 8}$, 1284-1287 (2016).

74. Purcell, S. et al. PLINK: a tool set for whole-genome association and population-based linkage analyses. Am. J. Hum. Genet. 81, 559-575 (2007). 


\section{ACKNOWLEDGEMENTS}

This research was conducted using data from the UK Biobank resource (application number 25331). Data collection for the Australian Genetics of Depression Study was possible thanks to funding from the Australian National Health \& Medical Research Council (NHMRC) to NGM (GNT1086683). AC-G is supported by a UQ Research Training Scholarship from The University of Queensland (UQ). MER thanks the support of the NHMRC and Australian Research Council (ARC), through a NHMRC-ARC Dementia Research Development Fellowship (GNT1102821). GC-P is funded by an ARC Discovery Early Career Researcher Award (DE180100976).

\section{AUTHOR CONTRIBUTIONS}

MER and GC-P conceived and directed the study. AIC and LMG-M performed most of the statistical and bioinformatics analyses, with support from GC-P and MER. EMB and NGM collected and contributed data from the Australian sample. AIC, LMG-M, MER and GC-P wrote the paper with feedback from all co-authors.

\section{COMPETING INTERESTS}

The authors declare no competing interests. 


\section{FIGURES AND TABLES}

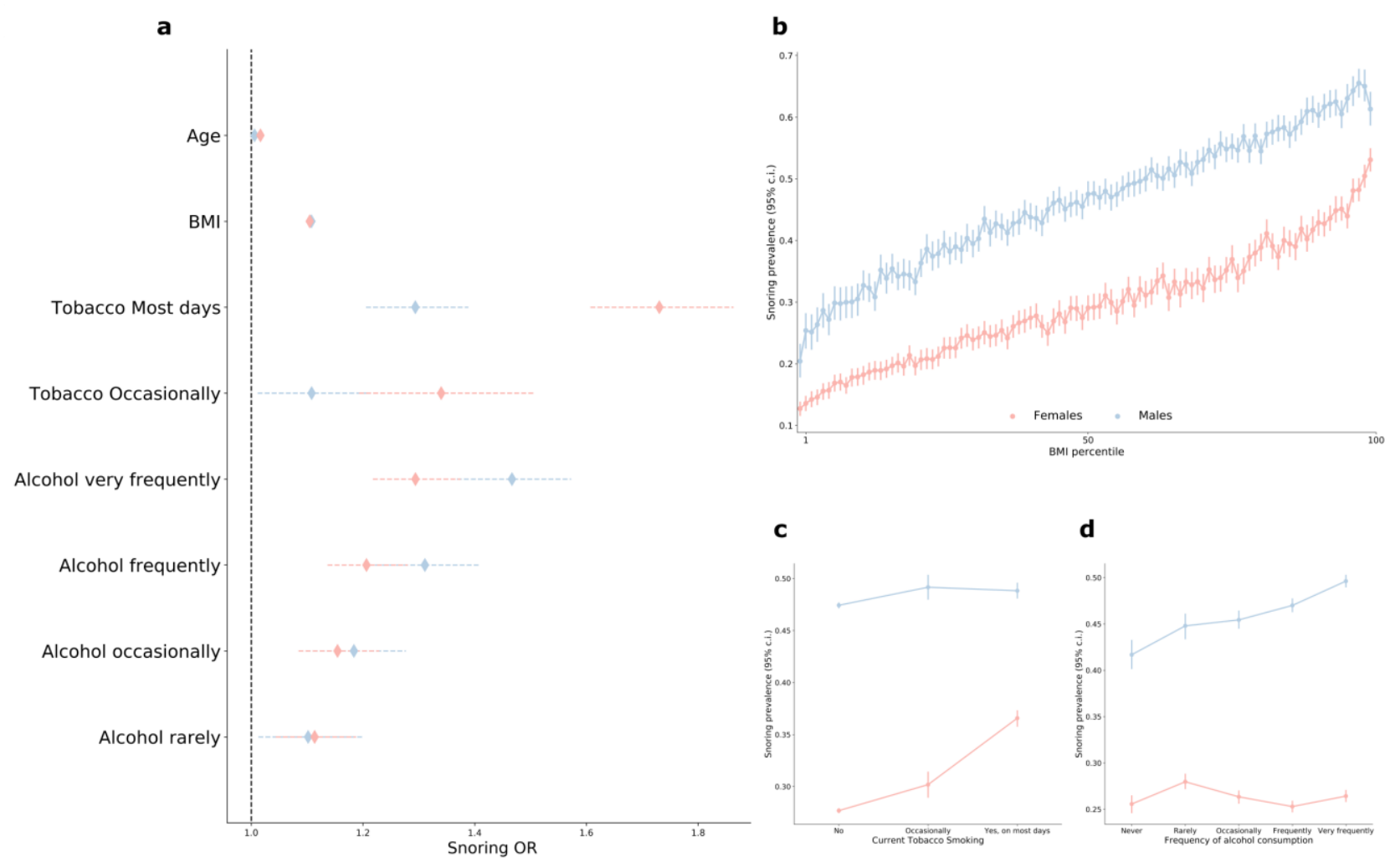

Figure 1. Sex, BMI, frequency of alcohol consumption and smoking are associated with increased snoring.

a) Forest plot depicting the odds ratios of studied variables on snoring for males (blue) and females (red). b) Sex and BMI stratified prevalence of snoring. c) Sex and frequency of tobacco smoking stratified snoring prevalence. d) Sex and frequency of alcohol consumption-stratified snoring prevalence. Error bars denote the $95 \%$ confidence intervals estimated using the odds ratio and standard error (a) or 1000 pseudo replicates from a bootstrap resampling procedure (b, c and d) 


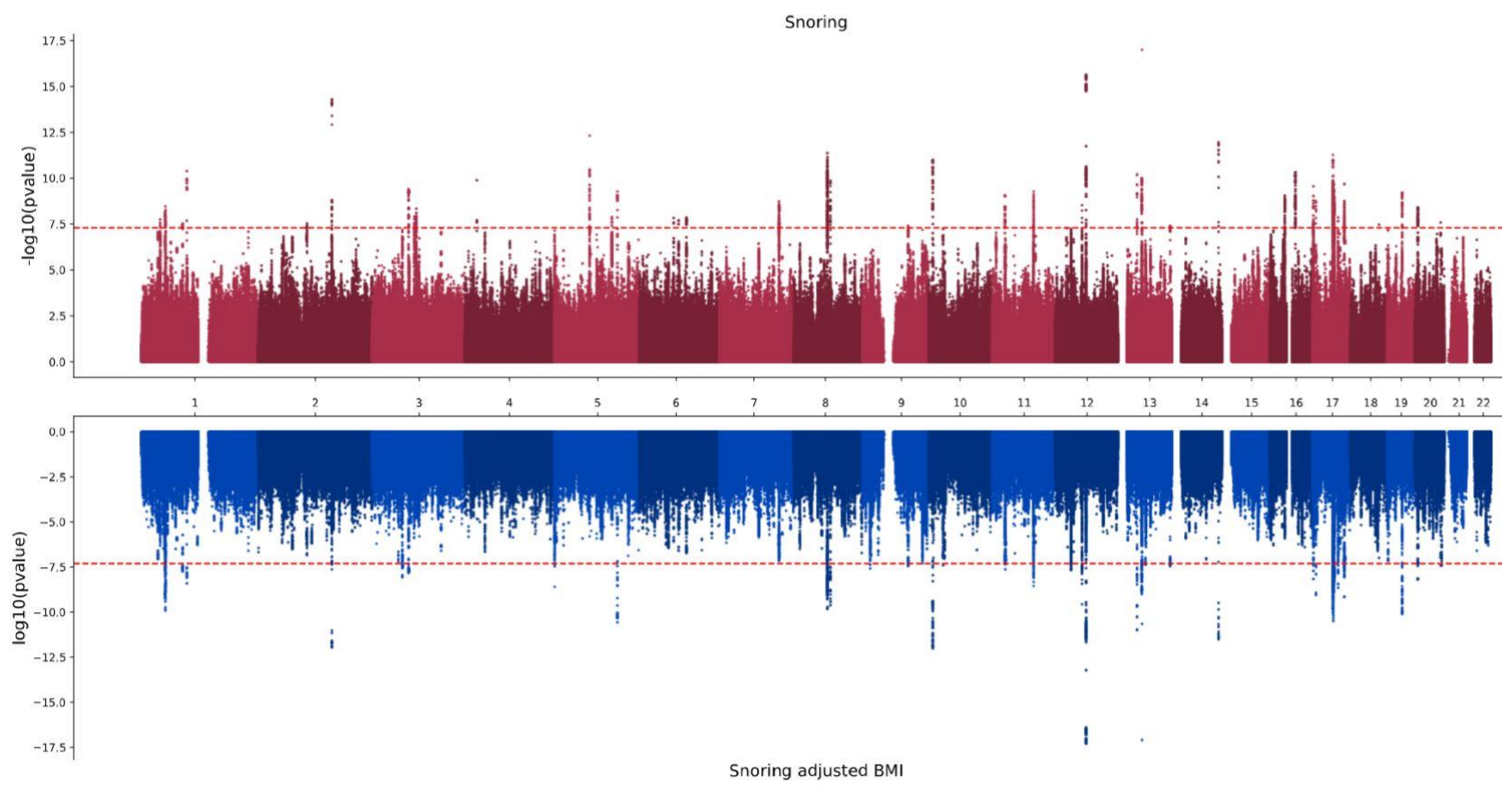

Figure 2. Genetic variants associated with snoring with (bottom) and without (top) adjustment for BMI.

Results from the genome-wide association studies are presented as mirrored Manhattan plots. The $\mathrm{x}$ axis contains a point for each genetic variant passing QC and is ordered by chromosome and base position. The distance between each variant and the $\mathrm{x}$-axis is a function of the significance ( $\mathrm{p}$-value) of the association. For the top panel the $-\log 10(\mathrm{p}$-value) is graphed on the y_axis whereas the $\log 10(\mathrm{p}$ value) is shown for the bottom panel. The red dotted line denotes the genome-wide significance threshold $(\mathrm{p}<5 \mathrm{e}-8)$. 


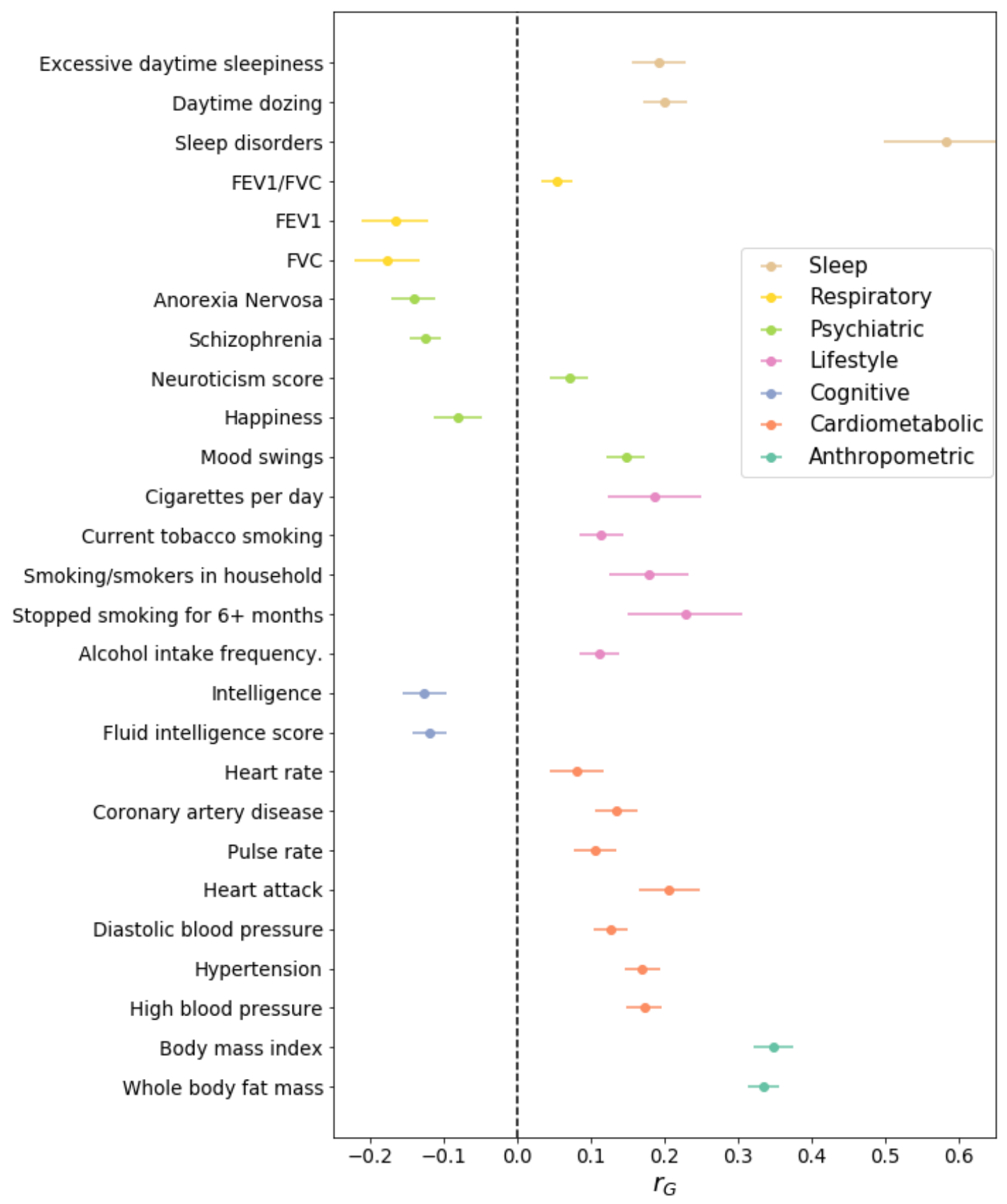

Figure 3. Snoring is genetically correlated with lifestyle, psychiatric, respiratory and other complex traits.

Circles represent LD score based estimates of the genetic correlation between snoring and other complex traits (y-axis). All of the depicted traits had a statistically significant genetic correlation with snoring after multiple testing corrections (benjamini-hochberg; fdr $<0.05$ ). Error bars show the s.e. Not all significant associations are shown due to lack of space, complete results are available in Supplementary File 2. 


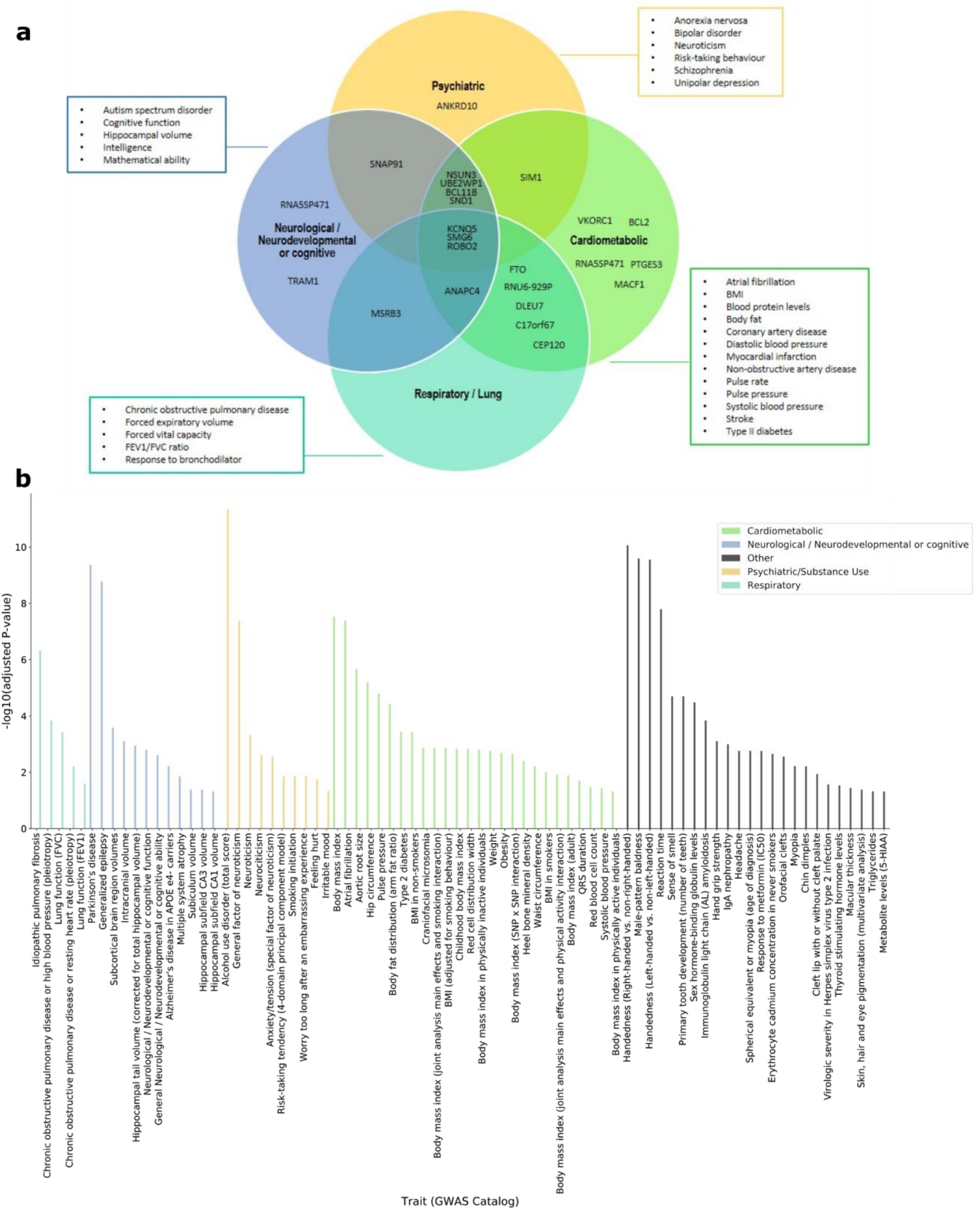

Figure 4. Snoring genes associated with other traits or diseases.

a) Venn diagram showing nearest genes to the lead significant SNP per genomic risk loci identified for snoring, categorised according to previously reported association with other traits or diseases in the GWAS catalog. b) Significant gene set enrichment analysis (hypergeometric test) based on all prioritised genes against gene-sets defined by traits in the GWAS catalog. 

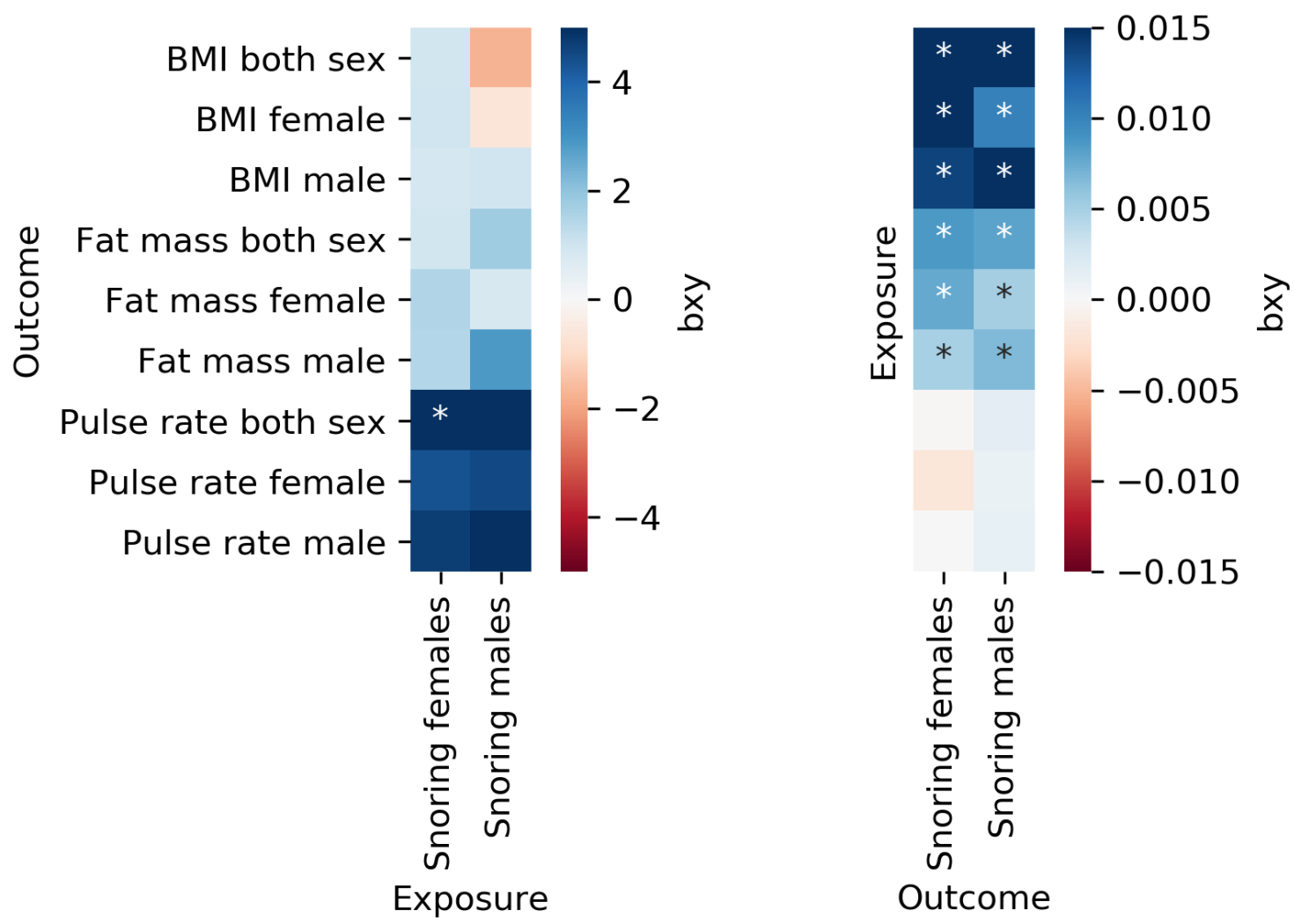

Figure 5. Mendelian randomisation suggests a one way causal relationship of BMI on snoring Heatmaps depicting Mendelian randomisation results testing for causal relationships between snoring and correlated variables. Colors represent the effect size and direction as estimated using GSMR. * denotes statistical significance after Bonferroni correction for the total number of MR tests performed $(\mathrm{p}<=1.39 \mathrm{e}-03)$. 

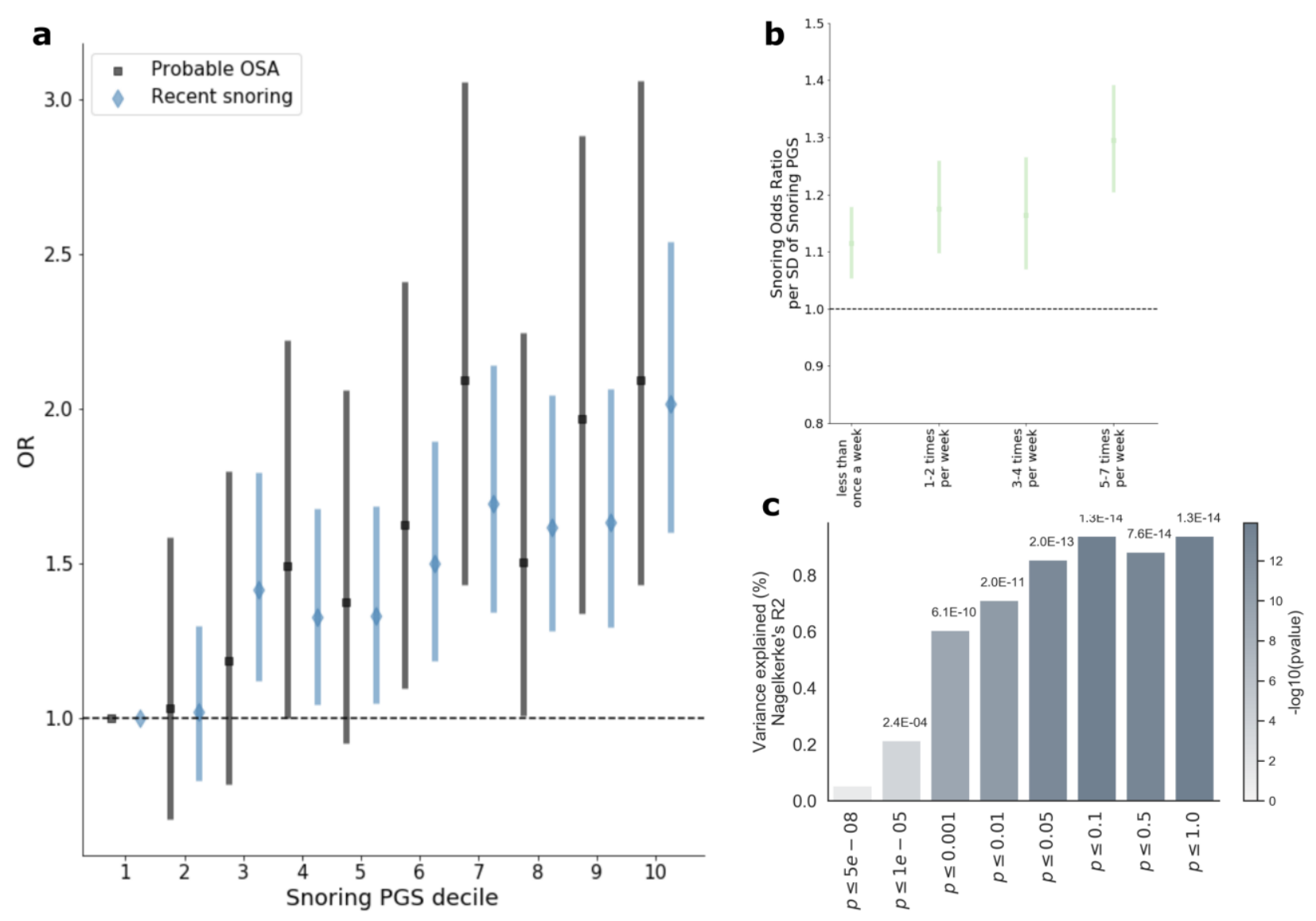

Figure 6. Polygenic scores predict snoring and probable apnoea in an independent sample

a) Forest plot showing the odds ratios (and 95\% CI) by decile of polygenic score (PGS) for snoring from the UK Biobank discovery sample (relative to the bottom decile $=1$ ) for recent snoring and probable sleep apnoea measured in an independent target sample of $\sim 8,000$ unrelated Australian adults from the Australian Genetics of Depression Study (AGDS). b) Snoring PGS predicting different snoring frequencies reported in AGDS target sample. c) Variance of recent snoring in AGDS explained by PGS calculated from UK Biobank summary statistics. The $\mathrm{x}$ axis represents the p-value threshold used for variant inclusion during genetic scoring; the y axis represents the amount of variance explained (change in Nagelkerke $\mathrm{R}^{2}$ ). The color of each bar represents the significance of the association between the PGS and recent snoring (-log10 p-value) while the exact p-value is shown above each bar. 
bioRxiv preprint doi: https.//doi org/10.1101/808691. this version posted October 17 2019. The copyriaht holder for this preprint (which was not certified by peer review) is the author/funder, who has granted bioRxiv a license to display the preprint in perpetuity. It is made available under aCC-BY-NC-ND 4.0 International license.

\section{Table 1. Sample composition and descriptive statistics of UK Biobank discovery sample}

\begin{tabular}{|r|c|c|c|c|}
\hline & Female N (\%) & Apnoea N (\%) & Age mean (sd) & BMI mean (sd) \\
\hline Cases (Snorers) & $63833(40.74 \%)$ & $4510(2.88 \%)$ & $57.01(7.70)$ & $28.67(4.85)$ \\
\hline Controls & $161775(61.44 \%)$ & $1663(0.63 \%)$ & $56.60(8.21)$ & $26.64(4.52)$ \\
\hline Total & $225608(53.72 \%)$ & $6173(1.47 \%)$ & $56.75(8.03)$ & $27.39(4.75)$ \\
\hline
\end{tabular}

$\mathrm{N}=$ Sample sizes. Descriptive statistics were calculated only for the subset of the data with european or british ancestry 
Table 2. Top 15 genomic risk loci for snoring showing the top SNP for each locus.

\begin{tabular}{|c|c|c|c|c|c|c|c|c|c|}
\hline SNP & Chr & Position & NEA & EA & MAF & Nearest gene & gwasP & Beta & SE \\
\hline rs592333 & 13 & 51340315 & G & A & 0.4423 & DLEU7 & $1.00 \mathrm{E}-17$ & -0.00906 & 0.001051 \\
\hline rs10878269 & 12 & 65791463 & T & C & 0.3499 & $M S R B 3$ & $2.30 \mathrm{E}-16$ & -0.00886 & 0.001086 \\
\hline rs61597598 & 2 & 156996626 & A & G & 0.1163 & $A C 073551.1$ & $5.10 \mathrm{E}-15$ & -0.01189 & 0.001529 \\
\hline rs2307111 & 5 & 75003678 & C & T & 0.3956 & POC5 & $4.80 \mathrm{E}-13$ & 0.007667 & 0.00107 \\
\hline rs2664299 & 14 & 99742187 & C & T & 0.4145 & BCL11B & $1.10 \mathrm{E}-12$ & 0.007503 & 0.001061 \\
\hline rs13251292 & 8 & 71474355 & G & A & 0.4145 & TRAM1 & $4.30 \mathrm{E}-12$ & -0.00737 & 0.001067 \\
\hline rs57222984 & 17 & 43758898 & G & A & 0.2654 & CRHRI:RP11-105N13.4 & $5.40 \mathrm{E}-12$ & -0.00843 & 0.00122 \\
& & & & & & ncRNA $)$ & & & \\
\hline rs725861 & 10 & 9063776 & G & A & 0.1918 & RP11-42L9.2 & $1.00 \mathrm{E}-11$ & -0.00908 & 0.001338 \\
\hline rs12119849 & 1 & 96878072 & A & G & 0.0825 & UBE2WP1 (pseudogene $)$ & $4.10 \mathrm{E}-11$ & -0.01226 & 0.00186 \\
\hline rs796856741 & 16 & 53799278 & GT & G & 0.4433 & FTO & $4.70 \mathrm{E}-11$ & -0.00696 & 0.001059 \\
\hline rs12429765 & 13 & 40745860 & G & A & 0.493 & LINC00332 & $6.20 \mathrm{E}-11$ & 0.0068 & 0.001051 \\
\hline rs34811474 & 4 & 25408838 & A & G & 0.2167 & $A N A P C 4$ & $1.30 \mathrm{E}-10$ & 0.007996 & 0.001237 \\
\hline rs7829639 & 8 & 78215352 & G & A & 0.2972 & $A C 105242.1($ miRNA $)$ & $1.40 \mathrm{E}-10$ & -0.00741 & 0.001155 \\
\hline rs180107 & 17 & 67930772 & T & A & 0.3698 & $A C 002539.2($ miRNA $)$ & $2.10 \mathrm{E}-10$ & -0.0068 & 0.00106 \\
\hline rs11409890 & 17 & 46269542 & TA & T & 0.4821 & SKAP1:RP11-456D7 & $2.20 \mathrm{E}-10$ & 0.006664 & 0.001061 \\
\hline
\end{tabular}


Table 3. SNP-based heritability of snoring on the liability scale.

\begin{tabular}{|l|c|c|c|c|}
\hline Trait & $\boldsymbol{h}^{2}$ SNP & SE & $\lambda_{\mathrm{GC}}$ & intercept \\
\hline Snoring & $9.9 \%$ & $0.39 \%$ & 1.4281 & $1.0427(0.011)$ \\
\hline Snoring adj. for BMI & $8.67 \%$ & $0.39 \%$ & 1.3685 & $1.0314(0.0094)$ \\
\hline Snoring males & $8.77 \%$ & $0.54 \%$ & 1.2005 & $1.0164(0.0078)$ \\
\hline Snoring females & $12.42 \%$ & $0.57 \%$ & 1.254 & $1.021(0.0074)$ \\
\hline Snoring adj. for BMI males & $7.72 \%$ & $0.56 \%$ & 1.2005 & $1.017(0.008)$ \\
\hline Snoring adj. for BMI females & $10.85 \%$ & $0.54 \%$ & 1.2531 & $1.0237(0.007)$ \\
\hline
\end{tabular}

LD score regression derived SNP based heritability results. Estimates were transformed to the liability scale assuming equal population and sample prevalences. $\lambda_{\mathrm{GC}}$ is the genomic inflation factor and intercept is the LD score regression intercept 
bioRxiv preprint doi: https://doi.org/10.1101/808691; this version posted October 17, 2019. The copyright holder for this preprint (which was not certified by peer review) is the author/funder, who has granted bioRxiv a license to display the preprint in perpetuity. It is made available under aCC-BY-NC-ND 4.0 International license.

\section{Supplementary file description}

Supplementary Materials. Supplementary Figures 1-6 and Supplementary Tables 1 - 4

Supplementary File 1. Genomic risk loci for discovery, sensitivity and sex-stratified analyses.

Supplementary File 2. FUMA output files for snoring and genetic correlations.

Supplementary File 3. FUMA output files for snoring adjusted for BMI and genetic correlations. 